\title{
PI5-0I. Willingness to participate in actual preventive HIV vaccine phase I/Ila trial in Bangkok, Thailand
}

\author{
A Hemachandra*1,2, RL Puls ${ }^{3}$, SJ Kerr ${ }^{3}$, N Saengthong ${ }^{1}$, K Pussadee ${ }^{1}$, \\ S Emery ${ }^{3}$, P Phanuphak ${ }^{1}$ and K Ruxrungtham ${ }^{1}$
}

Address: ${ }^{1}$ Medical department, HIVNAT, Thai Red Cross AIDS Research Centre, Bangkok, Thailand, Bangkok, Thailand, ${ }^{2}$ National Centre in HIV Epidemiology and Clinical Research, 376 Victoria Street, Australia and ${ }^{3}$ National Centre in HIV Epidemiology and Clinical Research, 376 Victoria Street, Australia

* Corresponding author

from AIDS Vaccine 2009

Paris, France. 19-22 October 2009

Published: 22 October 2009

Retrovirology 2009, 6(Suppl 3):P202 doi:10.1 186/1742-4690-6-S3-P202

This abstract is available from: http://www.retrovirology.com/content/6/S3/P202

(c) 2009 Hemachandra et al; licensee BioMed Central Ltd.

\section{Background}

Willingness to participate (WTP) in prophylactic HIV vaccine trials have been investigated amongst individuals at high risk for HIV and not in the actual HIV vaccine trial itself. We assessed the willingness and barriers to participation in an actual preventive HIV vaccine trial.

\section{Methods}

General population in Bangkok inquired about the HIV vaccine trial. Several questionnaires on HIV vaccine trial knowledge were completed during the recruitment process of phase I/IIa trial of DNA/Fowlpox vaccine. Self administered questionnaires on sexual HIV risk behavior, reasons and concerns to participate were completed at the end of the trial by participants and non-participants.

\section{Results}

From 131 participants who inquired, the median age was 34 years (IQR: $27-46$ ), 73\% were male and 30\% had at least a bachelor's degree and 11\% were screened for the trial. Only 34\% completed HIV vaccine knowledge questionnaires, the median score was not significantly different between those who came to screen and those who did not (median score was 7 (IQR $6-8$ ) and 6 (IQR 5 - 8) respectively; $\mathrm{p}=0.44)$. Baseline characteristics and method to recruit participants were not different between two groups. Of the $43 \%$ who completed self-administered questionnaires, those who were, male gender were more willing $(\mathrm{p}=0.038 ; \mathrm{OR}=6.29,95 \% \mathrm{CI}=0.75-52.68)$ by univariate analysis. Age and education were not associated with WTP. Knowing someone infected with HIV significantly decreased WTP $(\mathrm{p}=0.04 ; \mathrm{OR}=0.23,95 \% \mathrm{CI}=$ 0.05-1.01). The most common reasons volunteers declined to participate were concerns that their family would not want them to join and being not sure about the safety of the vaccine.

\section{Conclusion}

The low rate of actual WTP is common to HIV vaccine trials. Community education on the importance and safety of the vaccine is needed. 